ИЗВЕСТИЯ АКАДЕМИИ НАУК ЭСТОНСКОИ ССР. ФИЗИКА * МАТЕМАТИКА

PROCEEDINGS OF THE ACADEMY OF SCIENCES OF THE ESTONIAN SSR. PHYSICS * MATHEMATICS

$1987,36,3$

уДК 519.717 .3

Ю. ВАЙН

\title{
СРАВНЕНИЕ МОЩНОСТИ МОДЕЛИРОВАНИЯ МОДЕЛИ КЕРКА И СЕТЕЙ ПЕТРИ
}

\author{
(Представил Ю. Яаксоо)
}

Введение

В [ ${ }^{1}$ ] описывается формальная модель специфицирования и анализа систем взаимодействующих процессов - модель Керка (MK), которая принадлежит к классу систем с передачей сообщений. Введение количественного времени в МК с целью увеличения точности задания условий синхронизации и длительности взаимодействующих процессов является основным свойством, отличающим модель Керка от классических систем с сообщениями (см. $\left.\left[{ }^{2}\right]\right)$. Существенное увеличение мощности моделирования, связанное с введением параметров времени МK, поднимает вопрос о сохранении свойств разрешимости некоторых основных задач анализа и о точном месте $M$ K среди других формальных моделей параллельного вычисления. Для решения названных проблем необходимо сравнение MK с формализмом, для которого доказаны свойства разрешимости и мощности моделирования. Одним из наиболее изученных классов формальных моделей параллельного вычисления является класс сетей Петри (СП). Целью настоящей работы и является определение отношения между МК и ординарными СП.

\section{1. Метод сравнения}

Для формального сравнения МK с СП используем т. н. консервативный подход, описанный в $\left[{ }^{3}\right]$. Метод основан на нахождении преобразования, которое сопоставляет конструкциям одной модели конструкции другой так, что сохраняются структурные свойства и корректность поведения исходной модели.

О п р ед ел ен и е $1\left[{ }^{3}\right]$. Класс моделей $A$ равен по моциности моделирования классу В или меньше его, если для любого экземпляра а из класса $A$ существует алгоритм построения экземпляра $b$ из класса $B$, для которого выполняется следующцая совокупность признаков:

1) с т р укту рно е подоби е, т. е. каждая структурная компонента модели а представляется различным множеством компонент модели $b$; размер модели $b$ (число элементов) отличается от размера модели а не более чем на мультипликативную константу, причем константа определяется классами моделей $A$ u $B$, а не конкретными экземплярами а и

2) подоби е пов ед ения, т. е. любая последовательность действий в а может быть промоделирована их последовательностью в $b$; длина последовательности в $b$, отличается не более чем на мультипликативную константу от длины последовательности в а; 
3) сохранение некор ректностей, т. е. модель в заходит в тупик только тогда, когда заходит в тупик модель а (модель считается зашедшей в тупик, если все ее действия становятся невозможными).

Две модели $а$ и друг друга. Это предполагает, что любой экземпляр одной из моделей преобразуем в экземпляр другой модели.

Сопоставим теперь МK и СП, исходя из признаков сравнения, перечисленных в определении 1.

\section{2. Структурное подобие}

Описание элементов модели Керка. Основные понятия MK - процесс, канал, тип канала. Им можно сопоставить в первом приближении понятия СП, как это показано на рис. 1.
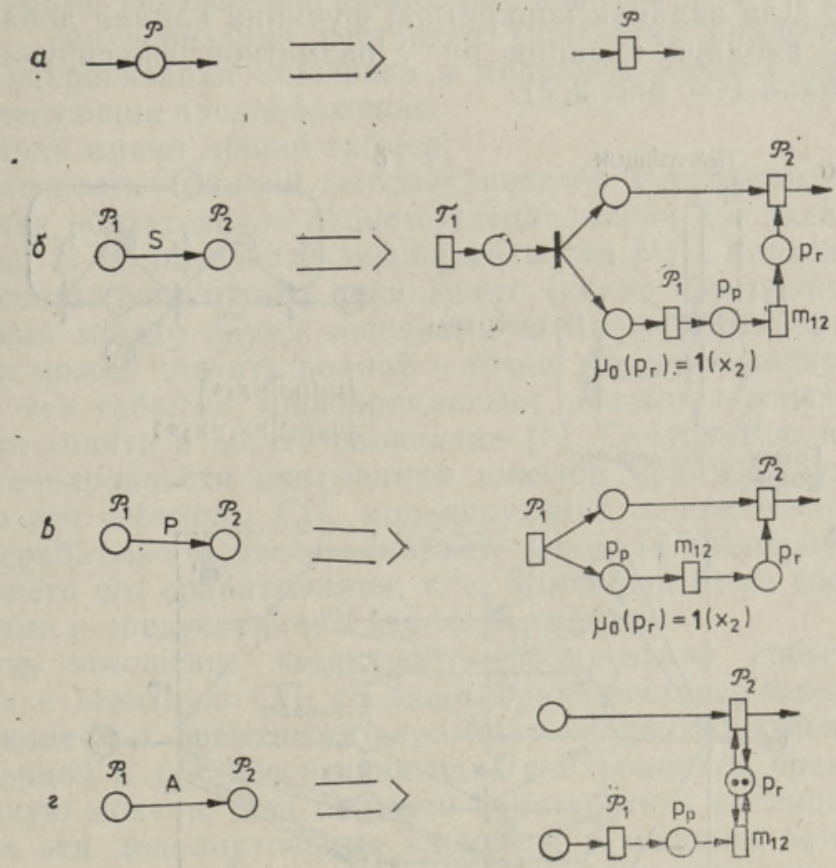

Рис. 1. Сопоставление элементов МК и СП. $a$ - процесс МК; $\sigma$ - синхронный канал MK; в - канал Петри MK; 2 - асинхронный канал MK $\left(m_{12}\right.$ - непримитивный переход, моделирующий функции канала; $\mu_{0}\left(p_{r}\right)$ - начальная разметка позиции $p_{r} ; 1\left(x_{2}\right)$ - единичная функция от аргумента функции канала MK).

Канал можно рассматривать концептуально как конечный FIFOбуфер, состоящий из двух частей с общим объемом $x_{1}+1$ (сохраняется $x_{1}+1$ последнего состояния процесса-поставщика). Предположим, что в функции канала $K\left(\sigma_{i j}, t\right)$, заданной в виде $\left[x_{1}, x_{2}\right]$, оба аргумента конечные натуральные числа $\left(x_{1}, x_{2} \in N\right.$ и $\left.0<x_{1}, x_{2}<\infty\right)$ и $x_{1} \geqslant x_{2}$. При затребовании данных из канала вычитывается $x_{1}-x_{2}+1$ самых старших состояний (см. рис. $2, a$ ).

Согласно такой концепции функцию канала можно моделировать с различной степенью детальности. Если ограничиться моделированием только операций записи в буфер, операций перемещения из одной части буфера в другую и вычитывания требуемых состояний, то сохранение постледовательности сообщений в канале на этой схеме в явном 
виде не представимо. С помощью разметки можно моделировать лишь число сохраняемых в канале состояний (см. рис. 2,6$)$.

Адекватная модель функции канала получится в случае, если моделировать занятость и незанятость каждой ячейки буфера и условия вычитывания из буфера, определенные только параметрами $x_{1}$ и $x_{2}$ значений состояния (см. рис. 2, 8 ). Для любых фиксированных значений $x_{1}$ и $x_{2}$ получим новую СП. Недостатком ее будет зависимость структурной сложности от конкретных значений функции канала. Предполагая все-таки, что параметры $x_{1}$ и $x_{2}$ конечные, т. е. меньше заданного целого числа $z$, мы можем конструировать конечное множество таких ограниченных сетей $C^{\prime m}$, где представлены все возможные комбинации параметров при условни $x_{1} \geqslant x_{2}$. Общее количество таких комбинаций

$$
C=\sum_{i=1}^{z} \quad i=z(z+1) / 2
$$

где $x_{1}, x_{2}<z$. Для задания конкретной функции канала добавим к каждой сети $C_{m}^{\prime}$ входную позицию $\mathrm{p}^{p \prime \prime}$ с разметкой, фиксирующей точную функцию канала (см. рис. 2, г).
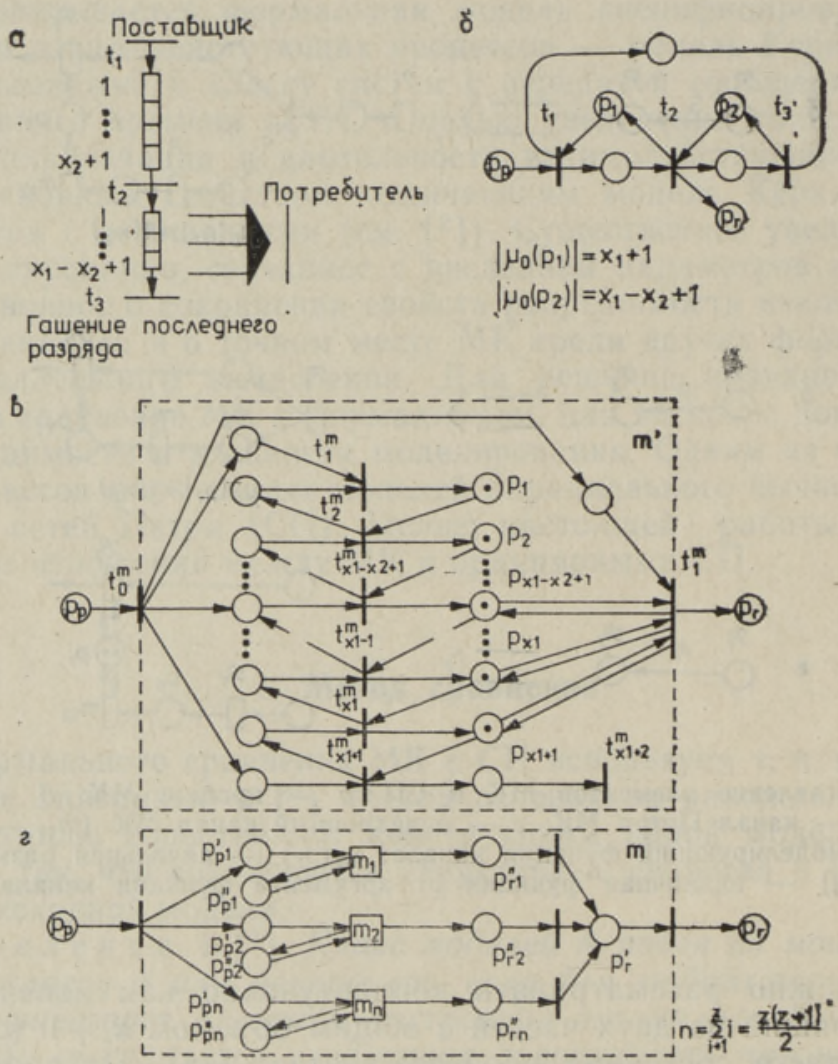

Рис. 2. Последовательность моделирования канала МК. $a$-концептуальная модель; 6 - неполная модель; $8-$ структурно зависимая модель $m^{\prime} ; 2-$ структурно независимая модель $m$.

Таким образом, моделирование параметров функции требует фактически проверки на нуль. Следовательно, в общем случае функция канала не представима структурно независимой СП, 
Моделирование временных параметров. Параметры времени в МК задают интервалы времени, в конце которых происходят определенные события. Поскольку в МK время дискретное, в конечном интервале существует конечное число временных отрезков - квантов. Для моделирования временных параметров с помощью СП будем рассматривать их как счетчиков, на вход каждого из которых поступают сигналы таймера, а на выходе счетчика сигнал появляется только после считывания необходимого числа тактов. После каждого запуска перехода в СП, моделирующей счетчик, работает только конечное, фиксированное число переходов. Следовательно, и множество всех достижимых состояний счетчика конечное. При этом условие выдачи выходного сигнала счетчика можно задавать только структурными средствами (напр., кратными дугами, см. рис. 3).

Как уже было показано при моделировании функции канала, структурно независимо СП, в принципе, можно построить толіько тогда, когда для всех временных параметров задана верхняя граница.

Однако кроме считывания временных квантов существует и другая проблема - синхронизация счетчиков (проблема единого времени в системе). Синхронизация счетчиков в модели возможна, если удовлетворяются следующие предположения:

- все счетчики имеют общий таймер;

- одновременность событий рассматривается с относительной точностью, т. е. как обязательное происхождение событий в заданном интервале времени (т. н. интервале эквивалентности $\left.\left[{ }^{4}\right]\right)$. Если длительность интервала составляет только один квант (квант определяется как отрезок времени между двумя соседними сигналами таймера), то одновременность можно считать полной с точки зрения моделирования. Это значит, что все события (одновременные) должны происходить после начала этого кванта и до его окончания [ $\left.{ }^{5}\right]$. Следовательно, при моделировании синхронности считывания квантов времени нужно обеспечить, чтобы все переходы СП, моделирующие одновременные события счетчиков, срабатывали после какого-то перехода таймера, но не позднее следующего его срабатывания, т. е. чтобы они были соответственно постусловиями и предусловиями перехода таймера.

Поскольку отношение частичного упорядочения событий хорошо описывается с помощью СП, то здесь принципиально возможно моделирование конечных временных параметров. Однако введение дополннтельных позиций и дуг, обеспечивающих синхронность, чрезвычайно осложняет такую модель. Для большей понятности и наглядности схем в дальнейшем эти дополнительные элементы в моделях приводиться не будут. Очевидно, что такие модели структурно зависят от значений параметров времени (см. рис. $3-5)$.

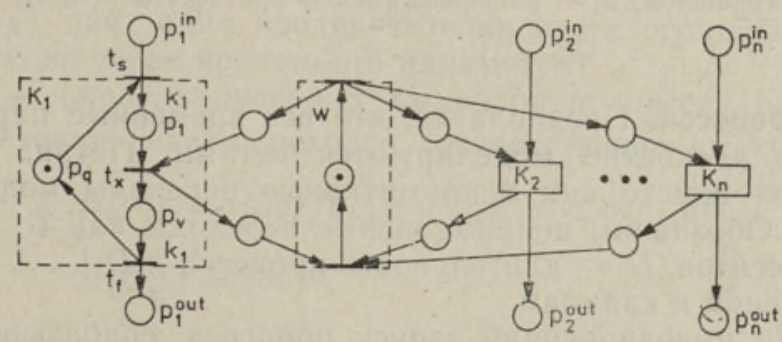

Рис. 3. Модель синхронного считывания временных квантов $\left(K_{1}, K_{2}, \ldots, K_{n}-\right.$ непримитивные переходы, моделирующие счетчнки длины $k_{i}, i=1, \ldots, n ; w-$ непримитивный переход, моделирующий таймер; $p_{i}^{\text {in }}, \ldots, p_{i}^{\text {out }}-$ позиции, моделирующие условия запуска и сигнализации $i$-го счетчика). 

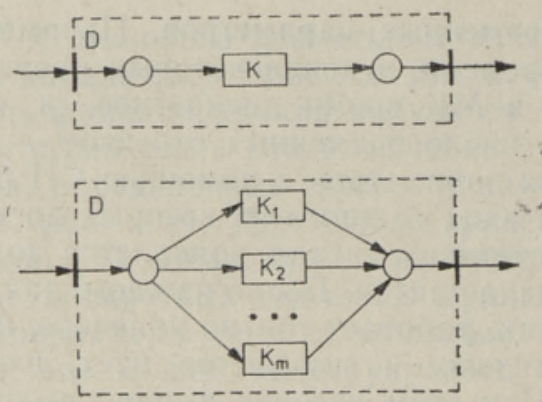

Рис, 4. Модели длительности процесса МК. a - длительность задана точно; 6 длительность задана с помощью конечного ннтервала.

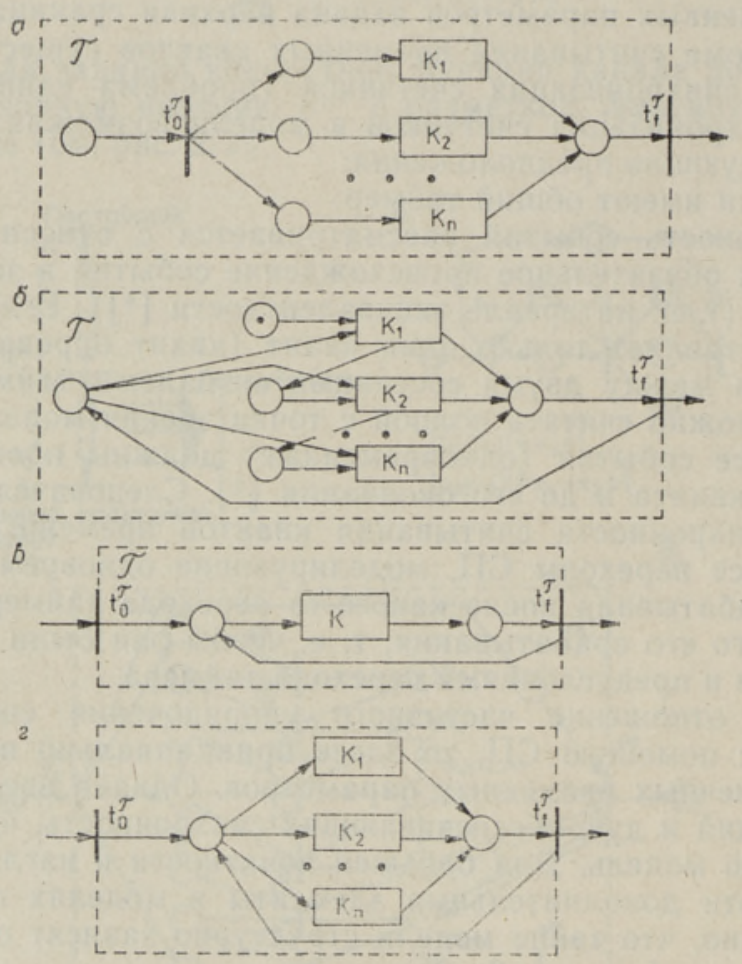

Рис. 5. Модели пусковых моментов МК; множество пусковых моментов задано: $a-$ в абсолютном времени, б - интервалами между моментами запуска, в - постоянным интервалом, $2-$ изменяющимся в промежутке $\left(t_{1}, t_{2}\right)$ интервалом.

Модель процесса. Предполагая, что все временные параметры и их синхронность адекватно моделируются сетями Петри, можем рассматривать их просто как непримитивные переходы модели процесса (см. рис. 6). Обозначим непримитивные переходы как $\mathfrak{C}$ - множество пусковых моментов, $D$ - длительность процесса и $D_{1}{ }^{I}, \ldots, D_{n}{ }^{I}-$ моменты обращений к каналам.

Переход $t_{s}$, моделирующй запуск процесса, срабатывает, когда из перехода $\mathcal{C}$ появляется фишка в позиции $p_{1}$. Если условия запуска заданы с помощью синхронных каналов или каналов Петри, фишка поступает из перехода $\mathcal{C}$ или $t_{f}$ процесса-поставщика. В результате работы перехода $t_{s}$ активизируются переходы $D, D_{1}^{\prime}, \ldots, D_{n}^{\prime}$. Для завер- 


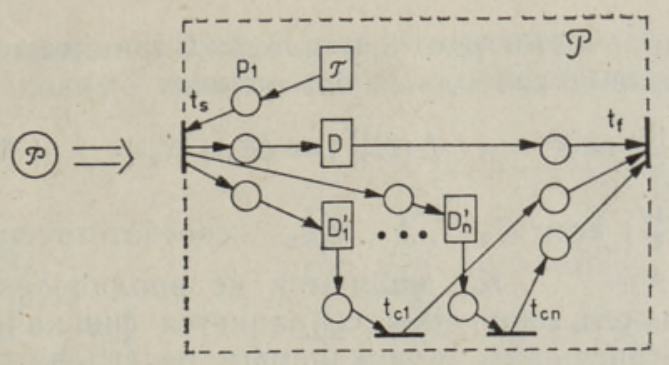

Рис. 6. Модель процесса МК.

шения работы процесса нужно, чтобы сработали переходы $t_{c 1}, \ldots, t_{c n}$, моделирующие получение сообщения из канала. Если хотя бы один из переходов $t_{c 1}, \ldots, t_{c n}$ не срабатывает, тогда процесс закончится не может.

\section{3. Подобие поведения}

Возможность моделирования отдельных элементов MK с помощью СП не означает, что это свойство сохраняется и при композиции элементов. Чтобы убедиться в замкнутости языков, полученных в результате композиции моделирующих элементов MK, мы должны показать, что при композиции используются только преобразования, сохраняющие тип языка СП. СП, моделирующие элементы МK, порождают следующее множество языков типа $L\left[{ }^{3}\right]$.

1. Сеть $C_{m}^{\prime}$ (модель функции канала, рис. 2,8 ).

Множество переходов: $T_{m}=\left\{t_{0}^{m}, t_{1}^{m}, \ldots, t_{x 1+1}^{m}, t_{x 1+2}^{m}, t_{f}^{m}\right\}$.

Алфавит: $\Sigma m=\{a, b, c, d, e\}$.

Функция помечения: $\quad \sigma: T_{m} \rightarrow \underset{m}{\Sigma}:\left\{\left(t_{0}^{m} ; a\right),\left(t_{x 1+1}^{m} ; b\right),\left(t_{x 1+2}^{m} ; c\right),\left(t_{x 1}^{m} ; d\right)\right.$, (без $\lambda$-переходов) $\left.\ldots,\left(t_{1}^{m} ; d\right),\left(t_{f}^{m} ; e\right)\right\}$.

Язык: $L\left(C_{m}\right)=\left(a b\left(c / / d^{m}\left(e / / d^{n}\right)\right)\right.$, где $m=x_{1}-x_{2}+1$ и $n=x_{2}$.

Язык $L\left(C_{m}\right)$ дает конечное множество последовательностей $(m, n<\infty)$.

2. Сеть $C_{K}$ (модель счетчика, рис. 3 ).

$$
\begin{gathered}
T_{K}=\left\{t_{s}, t_{x}, t_{f}\right\}, \\
\Sigma_{K}=\{a, b, c\}, \\
\sigma: T_{K} \rightarrow \Sigma_{K}:\left\{\left(t_{s} ; a\right),\left(t_{x} ; b\right),\left(t_{f} ; c\right),\right. \\
L\left(C_{K}\right)=a b^{k} c .
\end{gathered}
$$

Язык $L\left(C_{K}\right)$ дает одну последовательность с ограниченной длиной ( $k$ - конечное значение временного параметра).

3. Сеть $C_{\mathfrak{\tau}}$ (модель пусковых моментов процесса, рис. 5). При функции помечения без $\lambda$-переходов соответственно языки:

$$
\begin{aligned}
& L\left(C_{\tau}^{I}=a(b c / / b c)^{n}, \quad \text { где } n<\infty, \quad\right. \text { и } \\
& L\left(C_{\overparen{乙}}^{\text {II }}\right)=(b c)^{n},
\end{aligned}
$$

если $T_{\succ}=\left\{t_{0}^{\tau}, K_{1}, \ldots, K_{n}, t_{f}^{\complement}\right\}$,

$$
\Sigma_{\mathcal{C}}=\{a, b, c\} \text {, }
$$

$\sigma: T_{\mathcal{\zeta}} \rightarrow \Sigma_{\mathcal{\zeta}}:\left\{\left(t_{0}^{\mathcal{C}} ; a\right),\left(K_{1} ; b_{1}\right), \ldots,\left(K_{n} ; b_{n}\right),\left(t_{f}^{\mathfrak{C}} ; c\right)\right\}$, 
определяются вариантами рис. $5, a$ и 5 , б. Вариантами рис. 5, в и 5 , определяются языки со свободным помечением

$$
L\left(C_{\mathcal{C}}^{\mathrm{III}}\right)=K^{*} \quad \text { и } \quad L\left(C_{\mathcal{G}}^{\mathrm{IV}}\right)=\left(K_{1}+K_{2}+\ldots+K_{m}\right)^{*} .
$$

Хотя при $L\left(C_{\mathcal{C}}^{\mathrm{IV}}\right)$ сети $C_{K 1}, \ldots, C_{K n}$, соответствующие непримитивным переходам $K_{1}, \ldots, K_{n}$, являются не вполне заканчивающимися (в позициях $p_{q}$ после окончания сохраняется фишка см. рис. 3), тем не менее из-за конечности порождаемых последовательностей языки $L\left(C_{K}\right)$ с функцией свободного помечения являются замкнутыми по отношению к подстановке в бесконечную конкатенацию. Последнее обстоятельство особенно важно с точки зрения разрешимости задач анализа MK.

4. Сеть $C_{D}$ (модель длительности процесса, рис. 4, б).

$$
\begin{aligned}
& T_{D}=\left\{t_{0}^{D}, k_{1}, \ldots, k_{m}, t_{f}^{D}\right\}, \\
& \Sigma_{D}=\left\{a, b_{1}, \ldots, b_{m}, c\right\}, \\
& \sigma: T_{D} \rightarrow \Sigma_{D}:\left\{\left(t_{0}^{D} ; a\right),\left(K_{1} ; b_{1}\right), \ldots,\left(k_{m} ; b_{m}\right),\left(t_{f}^{D} ; c\right)\right\}, \\
& L\left(C_{D}\right)=a\left(b_{1}+\ldots+b_{m}\right) c, \quad m<\infty .
\end{aligned}
$$

5. Моменты обращений к каналу.

Языки порождаются так же, как и языки сетей $C_{D}$.

6. Сеть $C_{P}$ (модель процесса, рис. 6).

Сеть $C_{P}$ порождает язык со свободным помечением:

$$
L\left(C_{P}\right)=t_{s}\left(D / / D_{1}^{\prime} t_{c 1} / / \ldots / / D_{n}^{\prime} t_{c n}\right) t_{f}
$$

7. Процессы с различными типами каналов (рис. 1 и 6) порождают при композиции следующие языки:

синхронный канал: $\left.L\left(C_{s}\right)=\mathfrak{T}_{1} m_{12} / / \mathfrak{T}_{2}^{\prime}\right) \mathfrak{T}_{2}^{\prime \prime}$,

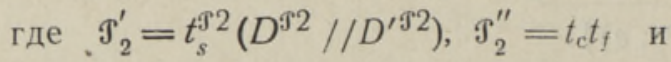

$m_{12}$ - непримитивный переход, моделирующий функции канала $\sigma_{12}$; канал Петри: $L\left(C_{P}\right)=\mathfrak{T}_{1}\left(m_{12} / / \mathfrak{T}_{2}^{\prime}\right) \mathfrak{T}_{2}^{\prime \prime}$;

асинхронный канал: $L\left(C_{A}\right)=\mathfrak{C}_{1} \mathfrak{T}_{1} m_{12} / / \mathscr{C}_{2} \mathfrak{T}_{2}$,

где $\mathfrak{G}_{1}$ и $\mathfrak{G}_{1}$ - непримитивные переходы, моделирующие соответственно множества пусковых моментов процессов $\mathfrak{T}_{1}$ и $\mathfrak{T}_{2}$.

Поскольку в языках $L\left(C_{S}\right), L\left(C_{P}\right), L\left(C_{A}\right)$ использованы только операторы параллельной композиции и конкатенации, а языки СП замкнуты по отношению этих операторов $\left[{ }^{3}\right]$, то языки $L\left(C_{S}\right), L\left(C_{P}\right)$, $L\left(C_{A}\right)$ принадлежат к классу языков СП.

Теперь покажем, что замкнутость языков СП сохраняется не только при моделировании исходных понятий $M \mathrm{~K}$, но и при использовании правил композиции МK.

Правила композиции МК, обеспечивающие замкнутость языков СП, следующие:

1) МK содержит конечное число процессов и каналов;

2) для однозначного задания пусковых моментов процессов, все пусковые моменты должны быть заданы либо множеством $T(p)$, либо только одним неасинхронным каналом;

3) из одного процесса может исходить несколько разных каналов, но в один синхронный кластер (синхронный кластер - совокупность процессов, связанных только с неасинхронными каналами $\left.\left[{ }^{4}\right]\right)$ не могут входить одновременно синхронный канал и канал Петри; 
4) перевычисляемые в результате выполнения процесса состояния поступают на выходные каналы всегда одновременно (отсутствует селективность).

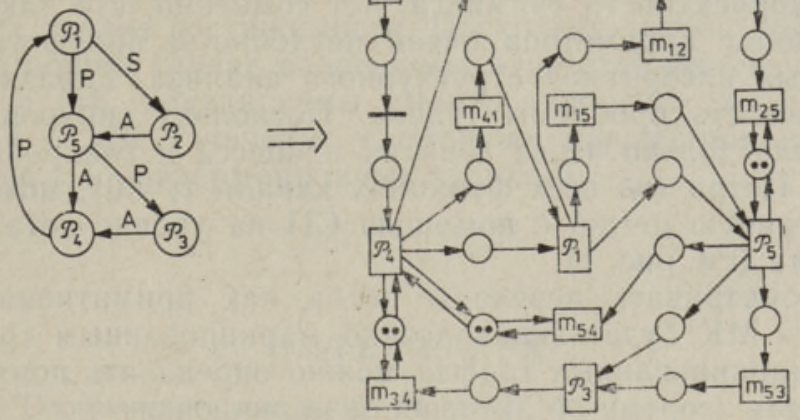

Рис, 7. Сопоставление МК и СП.

Для того чтобы определить свойства замкнутости языков MK, рассмотрим модель, построенную по всем приведенным правилам композиции (рис. 7). Модель генерирует язык $L(C)$ :

$$
\begin{aligned}
& L(C)=\mathscr{C}_{4} \mathfrak{T}_{4}\left(\mathfrak{T}_{2}^{\prime} / /\left(\mathfrak{T}_{1}^{\prime} / / m_{41}\right) \mathfrak{T}_{1}^{\prime \prime}\right)\left(m_{12} \mathfrak{S}_{2}^{\prime \prime} / /\right. \\
& \left./ /\left(\mathfrak{S}_{5}^{\prime} / / m_{15} \mathfrak{S}_{5}^{\prime \prime}\left(\mathfrak{S}_{3}^{\prime} / / m_{53} \mathfrak{S}_{3}^{\prime \prime}\right)\right)\right) .
\end{aligned}
$$

Язык $L(C)$ получен вследствие применения только следующих операторов: конечная подстановка, конкатенация, параллельная композиция и объединение. По отношению к таким операторам языки СП замкнуты $\left[{ }^{3}\right]$, и следовательно, MK, построенные по приведенным выше правилам композиции, являются также подклассом СП.

\section{4. Сводимость задач анализа корректности}

Возможность адекватного представления экземпляра MK и его функционирования с СП позволяет сформулировать теоремы корректности MK в терминах СП. Поскольку значения временных параметров заданы в моделирующих сетях с кратностью входных дуг позиции $p_{t}{ }^{K}$ (см. рис. 3), все проблемы корректности тайминга можно свести к задаче сравнения структурных свойств соответствующих сетей. Например, теорему о минимальном периоде запуска (см. [']']) можно переформулировать следующим образом:

Т ео рем а 1. Для того чтобы процесс $p\left(t_{i}\right)$ завершал работу раньше, чем $p\left(t_{i+1}\right)$, необходимо и достаточно, чтобы кратность входных дуг для позиций $p_{t}^{K 1 T}, p_{t}^{K 1 D}, p_{t}^{K n D 1}$ моделирующей СП была задана так, что

$$
\#\left(t, I\left(p_{t}^{K 1 t}\right)\right)>\#\left(t, I\left(p_{t}^{K m D}\right)\right)-\#\left(t, I\left(p_{t}^{k 1 D}\right)\right),
$$

где $p_{t}{ }^{K 1 т}$ - позиция $p_{t}($ подсети $K 1$ подсети $T)$, кратностью входных дуг которой задается минимальный интервал запуска процесса $\mathfrak{T}$;

$p_{t}{ }^{\text {ктD }}$ - позиция, кратностью входных дуг которой задается максимальная длительность процесса $\mathfrak{T}$;

$p_{t}{ }^{11 D}$ - позиция, кратностью входных дуг которой задается минимальная длительность процесса $\mathfrak{T}$; 
\# $(t, I(p))$ - функция, определяющцая кратность входных дуг позиции р, исходящих из перехода $t$.

Аналогично переформулируемы и теоремы 2 и 3 о корректности тайминга.

Несмотря на принципиальную возможность моделирования и анализа проблем корректности тайминга, нет сомнений, что такое представление временных параметров очень неудобно и предполагает применение сложных алгоритмов структурного анализа. Гораздо проще решается сводимость проблемы тупика. Поскольку синхронная петля в MK определена только через понятия процесса и синхронных каналов или каналов Петри (во всех функциях канала $x_{2}=0$ ), можно смоделировать синхронную петлю с помощью СП на уровне детальности процесса и канала (см. рис. 1).

Если рассматривать переходы петли как примитивные, то такое представление MK будет эквивалентно маркированным графам. Основываясь на маркированных графах можно определить понятие цикла и сформулировать теорему об активности маркировки.

О п р ед ел ен и е $2\left[{ }^{3}\right]$. Цикл в маркированном графе - это последовательность переходов $t_{j 1}, \ldots, t_{j k}$ такая, что для каждой пары переходов $t_{j r}$ u $t_{j r+1}$ из этой последовательности существует позиция $p_{i r}$ такая, ито $p_{i r} \in 0\left(t_{j r}\right) u p_{i r} \in I\left(t_{j r+1}\right)$.

т е о р м а $2\left[{ }^{3}\right]$. Разметка является активной тогда и только тогда, когда в каждом цикле маркированного графа присутствует по меньшей мере одна фишка.

По определению в СП, моделирующей $\mathrm{MK}$, фишка находится в выходных позициях $p_{r}$ (см. рис. 1) канала только тогда, когда второй аргумент функщии канала отличается от нуля. Если теперь фишки нет ни на одной из позиций, принадлежащих к циклу, т. е. предусловие теоремы 2 выполнено, то, согласно теореме 2, такая разметка является неактивной. Другими словами, все переходы $m_{i j}$, моделирующие функции канала, являются неактивными и никакая другая разметка, кроме нулевой, не достижима. Следовательно, задача анализа отсутствия тупика в МК сводима к задаче активности СП.

Другой подход к проблеме сводимости тупика МK приводится в работе $\left[{ }^{6}\right]$.

\section{Заключение}

На основе сравнения МK с СП можно сделать некоторые выводы о формальных свойствах МҚ.

1. Всякие попытки моделирования временных параметров $\mathrm{MK}$ с помощью СП приводили к проблеме проверки на нуль. Это проявлялось в том, что хотя модели элементов $M$ м можно было построить и все задачи анализа были в принципе, сводимыми, структурная независимость от параметров МК была получена только при их ограниченных значениях. Отсюда вывод, что только MK с ограниченными временными параметрами и функциями канала могут рассматриваться как подклассы СП.

2. МК моделирует маркированные графы в случае, когда все каналы типа Петри имеют функцию $[0,0]$, длительности процессов равны нулю и пусковые моменты заданы бесконечным интсрвалом [0, $]$. Но не все элементы МK моделируемы маркированными графами. Например, для моделирования случайного интервала запуска (см. рис. 5, 2) были использованы сети со свободным выбором.

3. Поскольку о внутренней структуре процессов МК не имеется никаких предположений, кроме того, что внутренняя структура процессов последовательна, с помощью МК нельзя моделировать конфликт- 
ность и выбор. Для устранения этого недостатка нужно зафиксироват̈ь язык описания процессов, где существуют нужные конструкции.

4. В принципе, проверка свойств корректности тайминга на СП не требует анализа достижимости и разрешима путем прямого сравнения кратности дуг определенных позиций. Только для обнаружения тупиков МК требуется анализ достижимости. Поскольку задачи достижимости и активности в общем случае разрешимы для сетей Петри, свободных от конфликтов, а МК моделируемы именно таковыми, то задачи тупика разрешимы и в МК (значения временных параметров не влияют на существование т. н. информационных тупиков).

\section{ЛИТ Е РАТ У РА}

1. Мытус Л. Л. Программирование, 3, 46-54 (1983).

2. Hoar, C. A. R. Commun. ACM, 21, 666-677 (1978).

3. Peterson, J. L. Petri Net Theory and the Modelling of Systems. New York, PrenticeHall, 1981.

4. Motus, L. Proc. of 6th IFAC Workshop on DCCS. 1985, 121-128.

5. Benmaiza, M., Thomesse, J. P. Preprints of IFAC/IFIP Symposium on RT Digital Contr. Appl. Mexico, 1, 1983, 64-72.

6. Текко Я. Изв. АН ЭССР. Физ. Матем. (1987) (в печати).

Институт кибернетики

Академии наук Эстонской ССР
Поступила в редакцию 17/XII 1986

\section{J. VAIN}

\section{QUIRKI MUDELI JA PETRI VÕRUDE VÕRLLUS MODELLEERIMISVŌIMSUSE JÄRGI}

On leitud tõkestatud parameetritega Quirki mudeli teisendus harilikeks Petri võrkudeks, mis säilitavad Quirki mudeli struktuuri ja dünaamika omadused. On näidatud, et tōkestatud parameetriga Quirki mudeli analüüsi probleemid on taandatavad Petri võrgu analüüsi probleemidele.

\section{COMPARISON OF QUIRK'S MODEL AND PETRI NETS, USING MODELLING POWER}

A partial transformation of Quirk's model to ordinary Petri nets has been defined. The transformation reduces certain analysis problems of the Quirk's model to those of Petri nets. 